\title{
Substrate specificity of proinsulin conversion in the constitutive pathway of transfected FAO (hepatoma) cells
}

\author{
F. Vollenweider, J-C. Irminger, P. A. Halban \\ Laboratoires de Recherche Louis Jeantet, University Medical Centre, Geneva, Switzerland
}

\begin{abstract}
Summary. Proinsulin is usually targetted to the regulated secretory pathway of beta cells, and converted to insulin in beta granules. Under certain pathological situations, a significant amount of proinsulin becomes diverted to the constitutive pathway. To study the kinetics of proinsulin conversion in the constitutive pathway, FAO (hepatoma) cells, which secrete proteins uniquely via this pathway and not the regulated pathway, were stably transfected with cDNA encoding human, rat I or rat II proinsulin. Products released to the medium of transfected cells were analysed by reversed phase HPLC and radioimmunoassay. For human proinsulin, des 31,32 split proinsulin (the conversion intermediate resulting from cleavage only at the B-chain/C-peptide junction followed by trimming of $\mathrm{C}$-terminal basic residues by carboxy-
\end{abstract}

peptidase) was the only detectable conversion intermediate; for rat proinsulin II it was des 64,65 split proinsulin (cleaved and trimmed only at the C-peptide/A-chain junction); for rat proinsulin I, both intermediates were seen. Complete processing to insulin occurred for all three, but was most extensive for rat proinsulin I. When considered with the corresponding proinsulin sequences, these data show that a -4 basic residue (i.e. 4 residues $\mathrm{N}$-terminal to the site of cleavage) facilitates proinsulin conversion in the constitutive pathway, and that arginine is preferred over lysine.

Key words: Constitutive secretion, prohormone processing, proinsulin, hepatoma (FAO cell).
In pancreatic beta cells, proinsulin is normally targetted to the regulated pathway and converted to insulin in secretory granules; less than $1 \%$ is secreted via the constitutive pathway [1]. However, under certain circumstances, inappropriate diversion of proinsulin to the constitutive pathway is of clinical significance (i.e. familial hyperproinsulinaemia, insulinomas) [2]. The fate of proinsulin in the constitutive pathway, however, remains poorly understood.

Until recently, it was thus not thought that this prohormone could be converted in the constitutive pathway. This pathway is not, however, enzymatically inert since it can entertain the conversion of several proproteins, proalbumin and the insulin proreceptor being two notable examples. In keeping with this conversion potential, we have recently reported [3] that some conversion of both human and rat II proinsulin can in fact occur in transfected hepatoma (FAO) cells, which are presumed to release proteins only via the constitutive pathway. We have now extended this study to include rat proinsulin I. The comparison of the primary sequence surrounding the sites of conversion in these three proinsulin molecules, when considered with the experimental results, indicates that proinsulin conversion in the constitutive pathway is strongly favoured by the presence of a -4 basic residue (i.e. 4 residues $\mathrm{N}$-terminal to the postulated point of cleavage) as indeed has been found for other proproteins converted in this pathway [4].

\section{Materials and methods \\ Transfection, cell culture and short-term incubation of FAO cells}

FAO cells (from Dr. C. Ronald Kahn, Joslin Diabetes Centre, Boston, Mass., USA) were cotransfected using the calcium phosphate method with pRSV-rInsI (rat I cDNA driven by the RSV LTR promotor) and pRSV-Neo [3]. Stably transfected clones were selected using G418 (2.5 $\mathrm{mg} / \mathrm{ml})$; they were grown in Dulbecco's modified Eagle's medium (DMEM) containing 10\% (volume/volume) fetal calf serum (FCS) and incubated in a humidified incubator at $37^{\circ} \mathrm{C}$ and $5 \% \mathrm{CO}_{2} / 95 \%$ air. Clones were further selected based upon their output of immunoreactive insulin (IRI).

For measuring the release of immunoreactive products, the cells (quasi confluent monolayers grown in 10-cm diameter petri dishes) were incubated for four sequential periods of $2 \mathrm{~h}$ in $10 \mathrm{ml}$ DMEM containing $0.5 \%$ bovine serum albumin (BSA); the media were pooled, and any remaining cells and debris were removed by cen- 
A

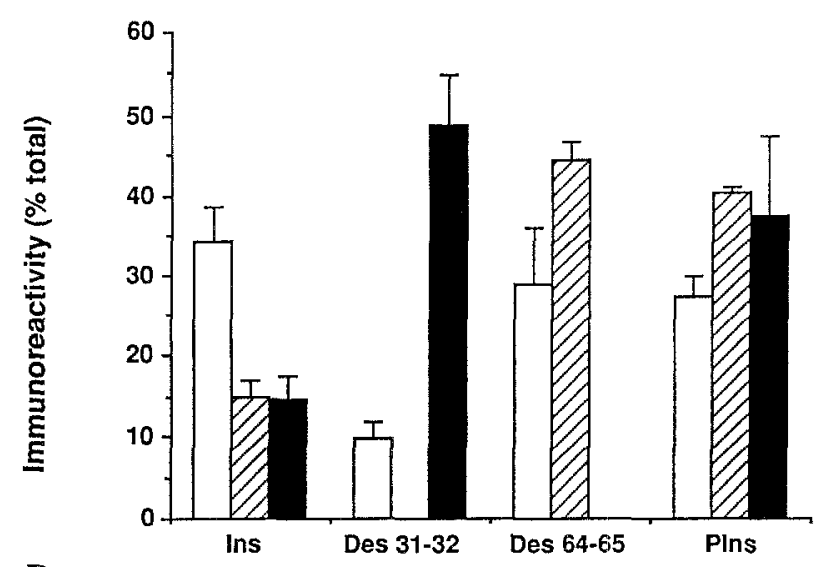

B

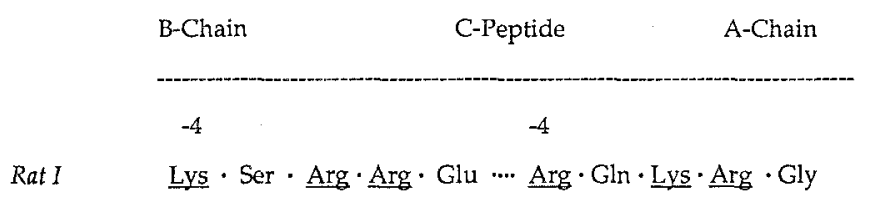

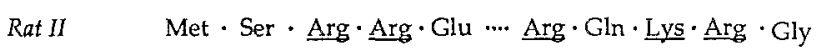

Human $\quad \underline{\text { Lys }} \cdot \mathrm{Thr} \cdot \underline{\mathrm{Arg}} \cdot \underline{\mathrm{Arg}} \cdot \mathrm{Glu} \cdot \ldots \mathrm{Leu} \cdot \mathrm{Gln} \cdot \underline{\mathrm{LyS}} \cdot \underline{\mathrm{Arg}} \cdot \mathrm{Gly}$

Fig. 1. A. Quantitative analysis of immunoreactive forms released to the medium from transfected FAO cells, Samples of medium were analysed by reversed phase HPLC. The immunoreactivity associated with insulin (Ins), des 31.32 or des 64.65 split proinsulin and proinsulin (PIns) is expressed as a percentage of the total immunoreactivity eluting from HPLC. The data are presented as mean + SEM for 2-3 independent observations. $\square$ rat I, $\square$ rat II, human. B. Sequence of human, rat I and rat II proinsulin at the B-chain/C-peptide and C-peptide/A-chain junctions. Basic residues are underlined and the position of the -4 residues shown above the sequences

trifugation. Cells were extracted in $1 \mathrm{~mol} / 1$ acetic acid, $0.1 \% \mathrm{BSA}$. The total insulin immunoreactivity released from the cells during this 8 -h period varied among clones, but was always in the range of 25 to $63 \mathrm{ng}$ per dish $\left(3-4 \times 10^{7} \mathrm{cells} / \mathrm{dish}\right)$.

The transfection of FAO cells with cDNA encoding rat II or human preproinsulin and the incubation conditions used for such transfected cells has been described in a previous report [3].

\section{HPLC analysis}

Medium and cell extracts were analysed by reversed-phase HPLC. Prior to injection, samples were prepurified and concentrated through C18 Sep-Pak cartridges, and insulin, proinsulin and conversion intermediates then separated by HPLC as described in detail previously [3]. For rat proinsulins and related products, the HPLC system was standardised by injection of radiolabelled material extracted from isolated rat islets that had been labelled for $60 \mathrm{~min}$ with $\left[{ }^{3} \mathrm{H}\right]$ Leu followed by a 60 -min chase. Radioactivity in each $1-\mathrm{ml} \mathrm{frac-}$ tion was measured by the addition of $3.5 \mathrm{ml}$ of Lumaflow II scintillation mixture (Lumac, Olen, Belgium). For human products, authentic standards (insulin, des 31.32 and des 64.65 split proinsulin, and proinsulin; the generous gift of Dr. R. Chance, Eli Lilly and Co., Indianapolis, Ind., USA) were used.

\section{Radioimmunoassay}

Insulin-related products were measured by radioimmunoassay. Rat (a mixture of rat insulin I and II) or human insulin standards as well as guinea pig anti-insulin serum were obtained from NovoNordisk (Bagsvaerd, Denmark). The cross-reactivity for human proinsulin or its conversion intermediates relative to native human insulin is approximately $50 \%$ on a weight basis and $75 \%$ on a molar basis.

\section{Results and Discussion}

Recently, it has become possible to measure with relative ease the circulating levels of proinsulin, conversion intermediates, and insulin in man [5]. The detection of unusually elevated levels of insulin precursors in individual patients could reflect one of a number of quite different pathological conditions. Characterising the molecular lesions responsible for such conditions and developing appropriate therapy will, however, depend upon a better understanding of proinsulin trafficking and processing in health and disease. In the beta cell, proinsulin is usually targetted with remarkably high efficiency to the regulated pathway [1], but on occasion such targetting may be less efficient [2]. One example would be the rare cases of familial hyperproinsulinaemia in which a mutation in the proinsulin molecule leads to its partial diversion to the constitutive pathway. A second example can be found in patients with an insulinoma; here the unusually elevated secretion of proinsulin-related products via a non-regulated pathway (presumably the constitutive pathway) has yet to be explained. The fate of proinsulin in the constitutive pathway is thus of considerable importance. Recently, we have shown that proinsulin can be processed in transfected hepatoma cells [3]. These cells are presumed to secrete proteins only via the constitutive secretory pathway and therefore provide an interesting experimental model system for following proinsulin processing in this pathway. We have now extended this study to allow for a quantitative comparison of the processing of three proinsulin molecules in the constitutive pathway (human, rat I and rat II).

Transfected FAO cells expressing proinsulin were incubated for 2-h periods and the incubation medium and cell extracts then analysed by reversed-phase HPLC. Insulin-immunoreactivity in each fraction eluting from HPLC was measured by radioimmunoassay. No conversion intermediates or fully processed insulin were found in cell extracts regardless of the molecular species of proinsulin expressed. HPLC analysis of the incubation media, by contrast, revealed interesting differences, as shown in Figure $1 \mathrm{~A}$. When the cells were transfected with rat insulin I cDNA, $34 \%$ of the immunoreactivity in the medium was insulin. Whereas both conversion intermediates were observed, des 64.65 split proinsulin predominated. For cells expressing human proinsulin, there was only $14 \%$ insulin, with close to $50 \%$ des 31.32 split proinsulin. There was no detectable des 64.65 split intermediate. Almost the exact opposite was found for rat proinsulin II, with $15 \%$ insulin, $45 \%$ des 64.65 split proinsulin and no detectable des 31.32 split proinsulin. 
Proinsulin is normally converted in secretory granules. Two endoproteases have been suggested to be implicated in such conversion. These enzymes are thought to correspond to PC1 (PC3) and PC2, two members of a family of mammalian subtilisin-like proteases related to the yeast Kex 2 gene product [6]. Expression of these two enzymes is restricted to cells with the regulated secretory pathway; cells which only release secretory proteins via the constitutive pathway but which are also able to convert proproteins must thus depend upon other endoproteases. Although it remains unclear precisely which enzyme is involved in proprotein processing in this pathway, other members of the mammalian Kex2-like family have been proposed, and notably furin and PACE4 [6]. These enzymes are most probably ubiquitous, but it is assumed that they will be limited in their activity to the constitutive pathway even of regulated secretory cells, with $\mathrm{PC} 1$ and PC2 active only in the regulated pathway, but this has yet to be demonstrated directly.

It has been shown that proprotein conversion in the constitutive pathway, by furin or a furin-like enzyme, depends upon the presence of a pair of basic residues at the site of cleavage. This is not, however, the only requirement. It has thus been found that for furin the consensus sequence for optimal conversion is R.X.K/R.R [7]. A survey of a number of proproteins converted in the constitutive pathway further shows that the majority display such a sequence with a basic residue in the -4 position relative to the site of cleavage, albeit with some notable exceptions [7].

When considered in the light of the present data, the comparison of the sequences surrounding the sites of cleavage of the two rat proinsulins and human proinsulin (Fig. 1B) reveals not only the impact on conversion of a basic residue in the -4 position, but also the relative competence of arginine or lysine. Consider first human proinsulin. As reported previously [3], although some insulin is released from FAO cells expressing this prohormone, des 31.32 split proinsulin is the major immunoreactive form detected in medium. This is proinsulin cleaved by endoprotease (and trimmed of C-terminal basic residues by carboxypeptidase) only between the B-chain and C-peptide, and its accumulation indicates facilitated cleavage at this site with only very limited cleavage at the C-peptide/A-chain junction. Whereas the former presents $a-4$ arginine, the latter has no -4 basic residue. For rat proinsulin II the opposite holds true [3]. The case of rat proinsulin I is perhaps the most interesting. Both cleavage sites present a -4 basic residue, and it is proposed that this accounts for the much higher levels of insulin found in the medium of cells expressing this proinsulin when compared with either rat II or human proinsulin. There was, furthermore, approximately twice as much des 64.65 split rat proinsulin I (cleaved and trimmed only at the C-peptide/A-chain junction) than des 31.32. Since the C-peptide/A-chain junction of this proinsulin presents a -4 arginine whereas the other junction has a -4 lysine, and assuming that there are no other constraints upon conversion at these two sites, we conclude that although either basic residue in the -4 position enables conversion to proceed, arginine is favoured over lysine. This means that the consensus sequence for proinsulin conversion in the constitutive pathway is similar to that for proproteins normally converted in this pathway, with R.X.R/K.R being preferred to K.X.R/K.R $[4,7]$. In keeping with this, it has been found that human proinsulin can be cleaved only at the B-chain/C-peptide junction by the convertase activity of isolated liver vesicles [8], and that rat proinsulin I can be converted to insulin in COS cells when cotransfected with furin [9]. It has furthermore been found that introduction of a tetrabasic motif (R.R.K.R.) at both cleavage sites in rat proinsulin I renders the prohormone more sensitive to cleavage by furin in COS cells [10]. Intriguingly, when native proinsulin is expressed in COS cells without the cotransfection of a conversion endoprotease, there is little or no processing [9]. Unprocessed proinsulin is also the major molecular form secreted via the constitutive pathway of other transfected cells such as L cells or Xenopus oocytes. The proinsulin conversion potential of the constitutive pathway of FAO cells may thus reflect an unusually high endogenous level of furin or a related conversion endoprotease.

Studying the behaviour of proinsulin in the constitutive pathway is of interest not only for gaining insight into the conversion machinery of this pathway per se, but also for detecting and understanding situations in which significant amounts of proinsulin have been diverted to this particular pathway. Assuming that proinsulin entering the constitutive pathway of the pancreatic beta cell is converted in much the same way as in FAO cells, we can thus now make certain predictions as to which products will be released by this pathway in cells expressing proinsulin of a given species.

Acknowledgements. This laboratory is a member of the Geneva Diabetes Group. We thank Ms. C.Caunes for expert technical assistance. This work was supported by Grant No. DK35292 from the National Institutes of Health and by a Grant from Hoechst AG.

\section{References}

1. Rhodes CJ, Halban PA (1987) Newly synthesized proinsulin/insulin and stored insulin are released from pancreatic $B$ cells predominantly via a regulated, rather than a constitutive, pathway. $\mathrm{J}$ Cell Biol 105: 145-153

2. Halban PA (1991) Structural domains and molecular lifestyles of insulin and its precursors in the pancreatic beta cell. Diabetologia 34: $767-778$

3. Vollenweider F, Irminger JC, Gross DJ, Villa-Komaroff L, Halban PA (1992) Processing of proinsulin by transfected hepatoma (FAO) cells. J Biol Chem 267: 14629-14636

4. Watanabe T, Nakagawa T, Ikemizu J, Nagahama M, Murakami K, Nakayama K (1992) Sequence requirements for precursor cleavage within the constitutive secretory pathway. J Biol Chem 267: 8270-8274

5. Sobey WJ, Beer SF, Carrington CA, et al (1989) Sensitive and specific two-site immunoradiometric assays for human insulin, proinsulin, 65-66 split and 32-33 split proinsulins. Biochem J 260: $535-541$

6. Seidah NG, Day R, Marcinkiewicz M, Benjannet S, Chrétien M (1991) Mammalian neural and endocrine pro-protein and prohormone convertases belonging to the subtilisin family of serine proteinases. Enzyme 45:271-284 
7. Hosaka M, Nagahama M, Kim WS, et al (1991) Arg-X-Lys/ArgArg motif as a signal for precursor cleavage catalyzed by furin within the constitutive secretory pathway. J Biol Chem 266: $12127-12130$

8. Brennan SO, Peach R.J (1991) The processing of human proinsulin and chicken proalbumin by rat hepatic vesicles suggests a convertase specific for X-Y-Arg-Arg or Arg-X-Y-Arg sequences. J Biol Chem 266: 21504-21508

9. Smeekens SP, Montag AG, Thomas G, et al (1992) Proinsulin processing by the subtilisin-related proprotein convertases furin, PC2, and PC3. Proc Natl Acad Sci USA 89: 8822-8826
10. Yanagita M, Nakayama K, Takeuchi T (1992) Processing of mutated proinsulin with tetrabasic cleavage sites to bioactive insulin in the non-endocrine cell line, COS-7. Febs Lett 311:55-59

Received: 1 June 1993 and in revised form: 9 July 1993

Dr. P.A. Halban

Laboratoires de Recherche Louis Jeantet

CMU, 1 rue Michel Servet

CH-1211 Geneva 4

Switzerland 